\title{
Using policy capturing to measure prejudicial attitudes of women toward women
}

\author{
ELIZABETH J. KATZ and JOSEPH M. MADDEN \\ Rensselaer Polytechnic Institute, Troy, New York
}

\begin{abstract}
To overcome the problem of obtaining only the socially desirable responses that often are elicited with direct methods, policy capturing was used as an indirect method of attitude measurement. A common direct method did not detect prejudicial attitudes, but policy capturing detected prejudicial attitudes in $37 \%$ of the female subjects in the study.
\end{abstract}

The purpose of this research was to test the hypothesis that women are prejudiced toward women. Research on this topic is limited because socially desirable responses are obvious and are easily given. Goldberg (1968) overcame this problem by having women read articles and asking them to judge how good the articles were. The only difference between identical articles was whether they were written by John McKay or Joan McKay. Goldberg found that in all cases the article authored by the male was rated more favorably than was the same article authored by the female. Other investigations have been sparse but have also produced findings that women are prejudiced toward women (Dipboye, Frankin, \& Witback, 1975; Fidell, 1970). The present study compared results obtained by a direct method of attitude measurement, in which socially desirable responses were easily given, with results obtained by an indirect method of attitude measurement, called policy capturing, in which socially desirable responses were impossible.

\section{METHOD}

\section{Subjects}

The subjects were groups of 92,98 , and 54 female students from a predominantly women's college.

\section{Procedure}

First, 92 subjects rank-ordered qualities of a Congressional candidate in terms of importance. The six qualities considered to be most important by the 92 subjects and used in the study were: honesty, knowledge of foreign and domestic affairs, understanding of constituents, knowledge of the law, intelligence, and leadership. Then, 98 subjects divided 100 points among seven qualities (the above six plus sex) to depict the ideal Congressional candidate. Thus, each subject provided an array of numbers, one for each of the seven qualities, so that the total of the numbers was equal to 100 . Each of these arrays can be

The authors' mailing address is: Department of Psychology, Rensselaer Polytechnic Institute, Troy, New York 12181.
Table 1

Examples of Medium Male and Female Profiles

\begin{tabular}{lcc}
\hline & $\begin{array}{c}\text { Pro- } \\
\text { file 1 }\end{array}$ & $\begin{array}{c}\text { Pro- } \\
\text { file 2 }\end{array}$ \\
\hline Honesty & 25 & 30 \\
Knowledge of foreign and domestic affairs & 10 & 10 \\
Understanding of constituents & 10 & 20 \\
Knowledge of the law & 10 & 10 \\
Intelligence & 20 & 25 \\
Leadership & 25 & 05 \\
Sex & M & F \\
\hline
\end{tabular}

considered to be a simulation or a profile of a Congressional candidate, with the magnitude of the number associated with each quality indicating the amount of the quality found in an ideal Congressional candidate, given that no candidate is perfect in terms of possessing a very high degree of all qualities. The mean number of points assigned to the sex of the candidate was 0 ; for all other qualities, the mean was 10 or more. Five of the 98 profiles were then selected randomly and designated as representative of a medium desirability level. Five high-level desirability profiles were constructed by doubling the values, and 5 low-level desirability profiles were formulated by halving the values, for a total of 15 profiles. Each of the 15 profiles was designated as male and again as female, making a total of $30 \mathrm{ex}-$ perimental profiles. An additional 23 profiles were selected randomly from the original 98 , and 12 were designated as female and 11 as male. The 53 profiles were then arranged randomly on a form and rated by all female subjects. The ratings were based on a 9-point scale, with 1 indicating a low degree of satisfaction with the individual simulated as a Congressional candidate and 9 indicating a high degree of satisfaction. Examples of a medium male and a medium female profile are given in Table 1.

\section{RESULTS}

An analysis of variance (sex $x$ level of desirability) yielded no significant Fs for a main effect of sex or interactions of sex with performance level.

A multiple regression equation was computed, using the subject's ratings as the dependent variable, for each of the 54 subjects. Five independent variables were generated by coding each profile for level, sex, and 
Table 2

Number of cases in 10-Point Intervals of $\mathbf{R}^{2}$

\begin{tabular}{rr}
\hline $\mathrm{R}^{2}$ & $\mathrm{~N}$ \\
\hline $11-20$ & 0 \\
$21-30$ & 3 \\
$31-40$ & 3 \\
$41-50$ & 6 \\
$51-60$ & 12 \\
$61-70$ & 19 \\
$71-80$ & 10 \\
$\geqslant 81$ & 1 \\
Total & 0 \\
\hline
\end{tabular}

interactions of sex and level. The subjects rated the profiles with a high degree of linear consistency, as shown by the distribution of $R^{2}$ in Table 2 .

The individual regression equations for each subject revealed that $20(37 \%)$ of the raters held prejudicial attitudes toward other females, as indicated by the entry of sex or of an interaction of sex and level into an individual's equation at the .05 level of significance.

\section{IMPLICATIONS AND CONCLUSIONS}

The results indicate that group statistics did not reveal prejudicial attitudes, whereas individual regression equations indicated that $37 \%$ of the subjects revealed prejudicial attitudes. This finding confirms the hypothesis that females are prejudiced toward females and that conventional sample statistics will not detect this prejudice.

The regression analysis method used in this study is called policy capturing. As has been shown, it is a powerful method in that it can be used to detect the degree of prejudice in a sample when that degree is too small to be detected by sample statistics. It has an additional strength in that it can be used on an individual basis as a diagnostic instrument.

\section{REFERENCES}

Dipboye, R. L., Frankin, H. L., \& Witback, K. (1975). Relative importance of applicant sex, attractiveness, and scholastic standing in evaluation of job applicants. Journal of Applied Psychology, 60, 39-45.

Fidell, L. S. (1970). Empirical verification of sex discrimination in hiring practices. American Psychologist, 25, 1094-1098.

GoldBERG, P. A. (1968). Are women prejudiced against women? Transaction, 5, 28-30.

(Manuscript received for publication October 31, 1983.) 